\title{
Fuzzy Bidirectional Weighted Sum for Face Recognition
}

\author{
Pengli Lu and Xingbin Jiang*
}

School of Computer and Communication, Lanzhou University of Technology, Lanzhou, 730050, China

\begin{abstract}
A new method for feature extraction and recognition, namely the fuzzy bidirectional weighted sum criterion (FBWSC) is proposed in this paper. FBWSC defines the row directional fuzzy image optimal image projection matrix. Subsequently, each sample in the original training sample set is transformed using the row directional optimal image projection matrix, and the row directional feature training sample set is obtained. Through the fuzzy distance, the row directional weight can be calculated. Similarly, FBWSC defines the column directional fuzzy image optimal image projection matrix; and then obtains the column directional feature training sample set. The column directional weight can be calculated using the fuzzy distance. Having obtained the row and column directional weight, FBWSC can sum the weight of row and column directional feature training sample sets, and then complete the feature extraction of the original sample data. Experiments on the ORL, FERET and Yale face database show that the proposed FBWSC method for face recognition has high recognition rate.
\end{abstract}

Keywords: face recognition, feature extraction, fuzzy bidirectional, fuzzy distance, weighted sum criterion.

\section{INTRODUCTION}

Face recognition is playing an increasingly significant role in public safety. Feature extraction based on dimensionality reduction is an important research topic in the field of computer vision and pattern recognition. The performance of dimensionality reduction directly decides the recognition rate of the algorithm. In the past few decades, principal component analysis (PCA) [1] and linear discrimination analysis (LDA) [2] are two of the most popular feature extraction algorithm.

As a powerful tool for data description, linear discrimination analysis (LDA) based on Fisher criterion function for feature extraction has been widely used in the field of pattern recognition and extension. However, it cannot be applied directly to small size sample (SSS) problem [3]. To address this problem, extensive methods, such as PCA+LDA [4], have been proposed in the literature. It is a two stage method, also known as the Fisherface method, in which PCA is first used for dimension reduction so as to make within-class scatter matrix $S_{W}$ nonsingular before the application of LDA. However, some useful discriminatory information may be lost. Li et al. [5] proposed maximum margin criterion (MMC). Unlike the LDA, MMC uses the difference between the within-class scatter matrix and between-class scatter matrix as a linear discriminate criterion. So, it does theoretically, fundamentally eliminates the problem in Fisher criterion because the within-class scatter matrix is singular. RB2DLDA [6] provides theoretical foundation for fusing class information in the two directions to design classifier and improve the recognition performance of the classifier.

In the PCA-based face recognition technique, the 2-D face image matrices must be previously transformed into 1-D image vectors. The resulting image vectors of faces usually lead to a high-dimensional image vectors space. 2DPCA [7] evaluates the image covariance matrix more accurately and computes the corresponding eigenvectors more quickly than PCA. RC2DPCA [8] can overcome the drawback of 2DPCA and select the optimal projection axis, which can compress image in row and column direction. RC2DPCA needs fewer coefficients for image representation than 2DPCA. However, the recognition rate of RC2DPCA is improved at the cost of computational time.

In this paper, Fuzzy Bidirectional Weighted Sum Criterion (FBWSC) is proposed for face recognition. The image matrix will be weighted fusion after row directional and column directional are compressed. Fig. (1) illustrates the entire FBWSC recognition procedure.

The organization of this paper is as follows: In section II, we briefly review the transformation in the row directional and column directional respectively. In section III, the idea of the proposed FBWSC method and the integration scheme are presented. In section IV, the performance boost of the proposed algorithm is analyzed via the time complexity. In section V, experiments with face images data are presented to demonstrate the effectiveness and superiority of the new method. Conclusions are summarized in section VI.

\section{TRANSFORMATION IN TWO DIRECTIONAL}

Given a set of data points $X=\left\{X_{1}, X_{2}, \cdots, X_{i}, \cdots, X_{L}\right\}$, the $i$ th class is $X_{i}=\left\{X_{i 1}, X_{i 2}, \cdots, X_{i j}, \cdots, X_{i n}\right\}$, where $L$ is the number of 


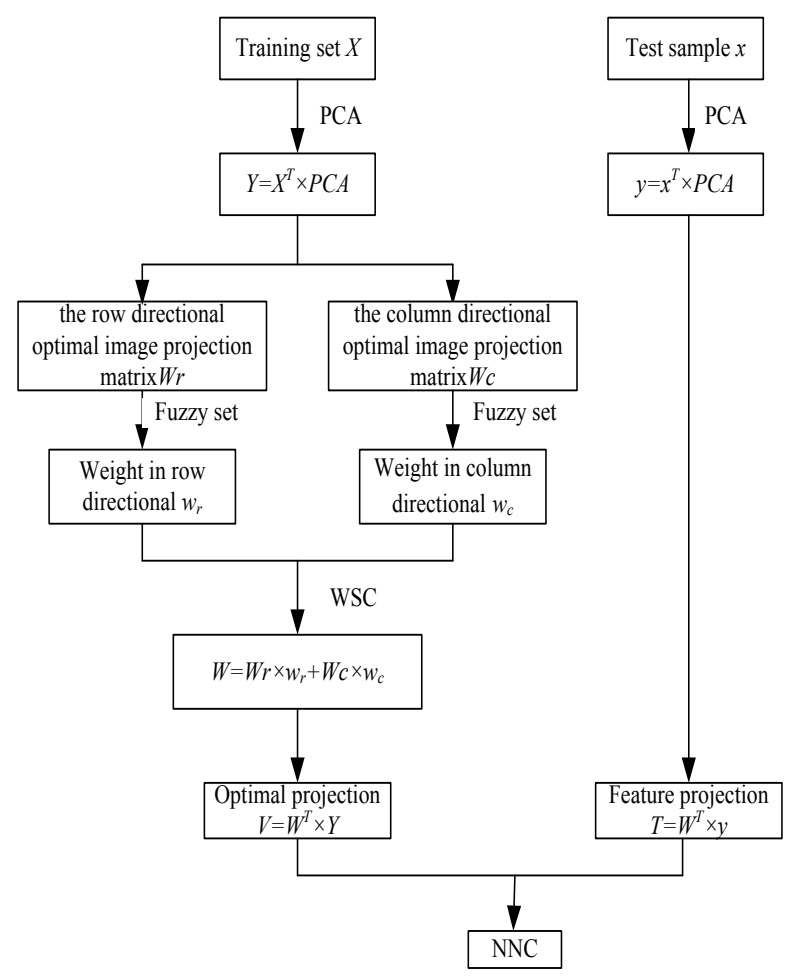

Fig. (1). Illustration of recognition procedure of FBWSC.

classes and $n$ is the number of samples in class $i . N=L \times n$ is the number of the total samples. $\bar{X}$ is the mean matrix of all training samples and $\bar{X}_{i}$ is the mean matrix of class $i$.

The row within-class scatter matrix $S_{w}^{r}$ is defined by $S_{W}^{r}=\sum_{i=1}^{L} \sum_{j=1}^{n} \frac{1}{N}\left(X_{i j}-\bar{X}_{i}\right)\left(X_{i j}-\bar{X}_{i}\right)^{T}$ and the row betweenclass scatter matrix $S_{B}^{r}$ is defined by $S_{B}^{r}=\sum_{i=1}^{L} \frac{n}{N}\left(\bar{X}_{i}-\bar{X}\right)\left(\bar{X}_{i}-\bar{X}\right)^{T}$. Then the transformation matrix in row direction $P_{\text {row }}$ derived by $\mathrm{P}_{\mathrm{row}}=\arg \max \frac{W^{T} S_{B}^{r} W}{W^{T} S_{w^{W}}^{r}}$.

Similarly, the column within-class scatter matrix $S_{w}^{c}$ is defined by $S_{W}^{c}=\sum_{i=1}^{L} \sum_{j=1}^{n} \frac{1}{N}\left(X_{i j}-\bar{X}_{i}\right)^{T}\left(X_{i j}-\bar{X}_{i}\right)$ and the column between-class scatter matrix $S_{B}^{c}$ is defined by $S_{B}^{c}=\sum_{i=1}^{L} \frac{n}{N}\left(\bar{X}_{i}-\bar{X}\right)^{T}\left(\bar{X}_{i}-\bar{X}\right)$. Then the transformation matrix in row direction $P_{c o l}$ derived by $\mathrm{P}_{c o l}=\arg \max \frac{W^{T} S_{B}^{c} W}{W^{T} S_{B}^{c} W}$.

The discriminant information of projection transformation in the row direction and column direction are con- sistent and complementary to each other [6]. It provides the theoretical basis for the fusion of the discriminant information on two directions and improves the recognition rate of the classifier.

\section{WEIGHTED INTRODUCTION}

The form of feature extraction is different from BDPCA $[9,10]$ and RC2DPCA, and their projecting matrices come from different theory.

In BDPCA, $Y=P_{c o l}^{T} \times X \times P_{\text {row }}, P_{\text {row }}$ and $P_{c o l}$ have no direct relation, they are just forcedly used to feature extract simultaneously. In RC2DPCA, $C=U^{T} Y=U^{T} \times X \times P, 2$ DPCA is implemented on the original image space $X$ to get the projecting matrix $P$, alternative 2DPCA is implemented on the feature space $Y^{\mathrm{T}}$ to get the projecting matrix $U$. Projecting image onto $P$ and $U^{\mathrm{T}}$ can compress image more efficiently in row and column directions.

The proposed Weighted Sum Criterion is to perform the row directional transformation and the column directional transformation weighted integration. $W=P_{\text {row }} \times w_{\text {row }}$ $+P_{c o l} \times w_{c o l}, w_{\text {row }}$ and $w_{c o l}$ are the weight on the row direction and the column direction respectively. Then the optimal projection of the original images can be rewritten as $Y=W^{T} \times X$.

The weights on the row and column direction have a great effect on the performance of FBWSC. We try to find out the complete distribution information of samples through 


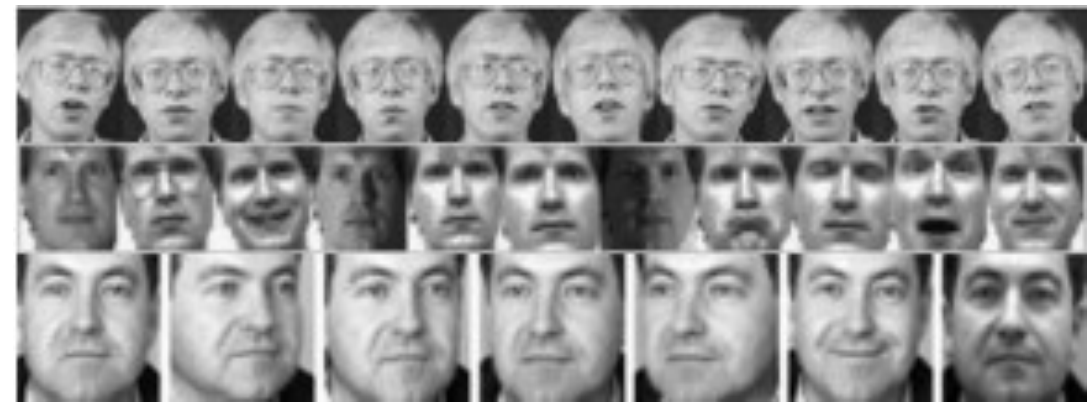

Fig. (2). The sample images of one person in three databases respectively. From top to bottom is in turn ORL, Yale and FERET database.

the fuzzy set theory. The sample distribution information is represented by fuzzy membership degree corresponding to each class. So we can make use of fuzzy membership degree to represent the different proportion of two directions. Our approach is to find out the fuzzy membership degree [11] in the change of the original images on row direction and column direction respectively firstly, then compute the mean fuzzy distances on two directions using the fuzzy membership degrees, normalization of fuzzy distances and as the weight in two directions in the end. The idea of weighted in FBWSC can be summarized below:

Step 1. According to the fuzzy k-nearest theory [12], calculate the distance between each other $D$ and the membership degree of the samples using (1).

$u_{i j}=\left\{\begin{array}{l}0.51+0.49 \times\left(n_{i j} / k\right), j \in i \\ 0.49 \times\left(n_{i j} / k\right), j \notin i\end{array}\right.$

Step 2. Compute the mean of fuzzy distance $s_{r}$, after performing row transformation using (2).

$s_{r}=\frac{1}{2 N(L-1)} \sum_{m=1}^{N} \sum_{i=1}^{L} \sum_{j=1}^{n} \mu_{i m} D_{j+(i-1) \times l, m}$

Similarly, the mean of fuzzy distance after performing column transformation can be computed as the same form.

Step3. The weight on row and column direction are $w_{\text {row }}=s_{r} /\left(s_{c}+s_{r}\right)$ and $w_{\text {row }}=s_{c} /\left(s_{c}+s_{r}\right)$.

Step4. The optimal projection matrix $W$ can be computed by $W=\mathrm{P}_{\text {row }} \times w_{\text {row }}+P_{c o l} \times w_{c o l}$, where $\mathrm{P}_{\text {row }}$ and $\mathrm{P}_{c o l}$ is illustrated in section II.

\section{TIME COMPLEXITY ANALYSIS}

The idea of the traditional bidirectional algorithms, such as RC2DPCA, FBMMC and RB2DLDA, are to perform the row directional transformation and the column directional transformation, sequentially. Then the calculation of the op- timal projection needs to order matrix multiplication twice. The time complexity is

$$
T([m \times m] \bullet[m \times n] \bullet[n \times n])=T(m \times m \times n \times n)=\mathrm{O}\left(n^{4}\right) .
$$

However, the idea of FBWSC is fusing the two directional projection matrices and obtaining the optimal projection. Obviously, it's time complexity is

$$
T([m \times m] \bullet[m \times n])=T(m \times m \times n)=\mathrm{O}\left(n^{3}\right) .
$$

According to the above analysis, the proposed FBWSC can reduce the computation time in theory compared with traditional algorithm.

\section{EXPRIMENTS AND DISCUSSION}

To evaluate the proposed FBWSC algorithm, three face image databases, namely, the ORL database, FERET database and Yale database are used to compare the proposed FBWSC approach with the following algorithms 2DLDA, RC2DPCA and FBMMC. The ORL database was used to evaluate the performance of FBWSC under conditions where the pose and sample size are varied The FERET face database involves variations in facial expression, illumination and pose. The Yale database was used to examine the system performance when both facial expressions and illumination are varied. In addition, the focus of this study is mainly on feature representation thus, in classification stage, Euclidean distance is employed to measure the similarity between two features and the nearest neighbor classifier for classification. In the PCA phase, we keep 98 percent image energy. The number of nearest neighbor $k=3$. Table 1 presents the top recognition rates of the methods and the corresponding dimension on three databases. Fig. (1) shows sample images of one person in three databases.

Table 1. The top recognition rates of four features extraction.

\begin{tabular}{|c|c|c|c|}
\hline & ORL & FERET & Yale \\
\hline \hline 2DLDA & $84.33 \% 22$ & $54.78 \% 18$ & $80.00 \% 18$ \\
\hline RC2DLDA & $85.21 \% 20$ & $57.89 \% 12$ & $81.67 \% 18$ \\
\hline FBMMC & $85.56 \% 16$ & $58.89 \% 14$ & $82.33 \% 16$ \\
\hline Proposed & $88.67 \% 6$ & $64.33 \% 8$ & $83.60 \% 10$ \\
\hline
\end{tabular}




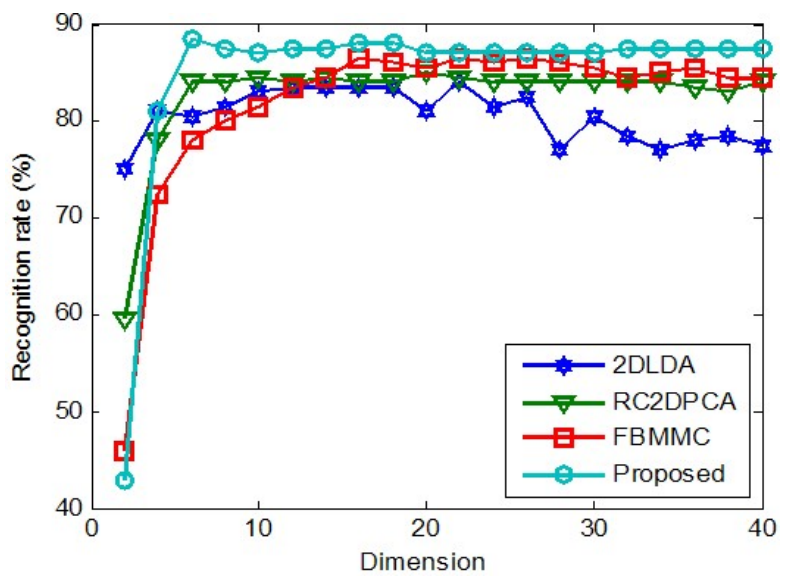

Fig. (3). Recognition rates versus dimensionality on the ORL face database when the first five images per class are selected for training.

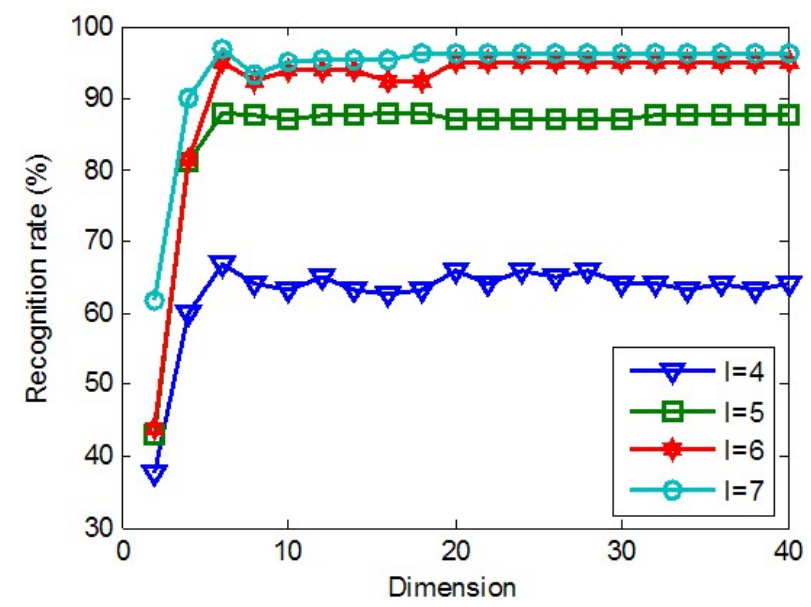

Fig. (4). Recognition rates over the variance of $l$ on ORL.

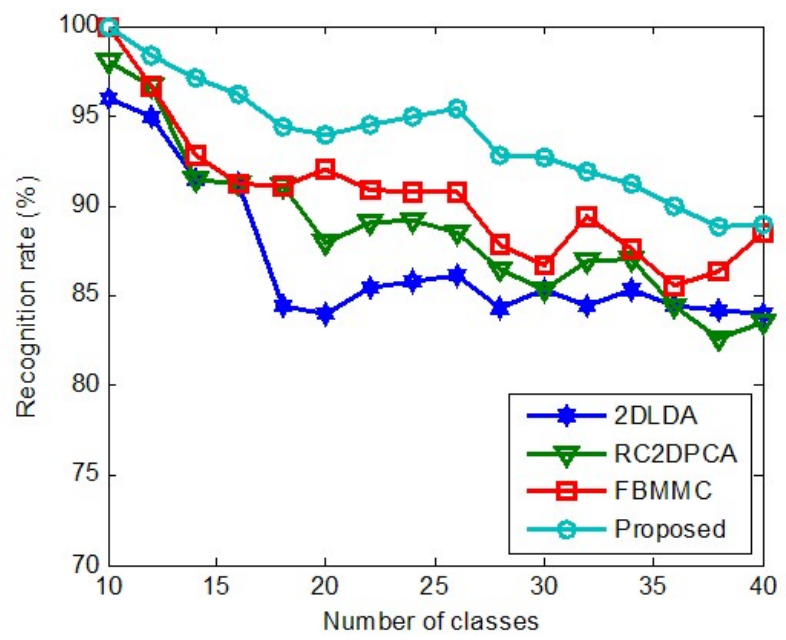

Fig. (5). Recognition rates over the variance of classes on ORL.

\section{A. Experiments on the ORL Database}

The ORL database [13] is composed of 40 individuals, each providing 10 different images under different time, dif-

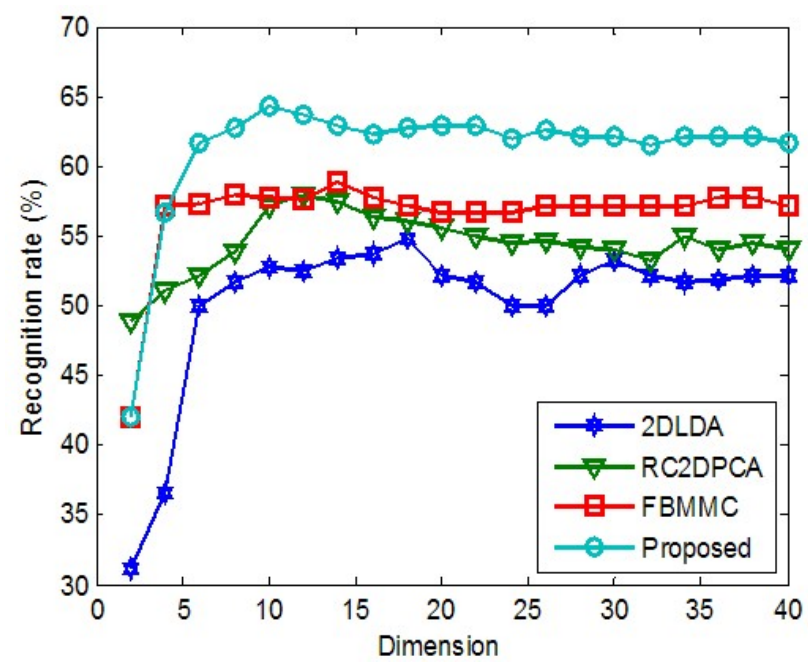

Fig. (6). Recognition rates versus dimensionality on the FERET face database when the first four images per class are selected for training.

ferent expression and different views, with a tolerance for some tilting and rotation of up to about 20 degrees. Some people are captured with or without glasses. Moreover, the heads in images are slightly tilted or rotated. The images in the database are manually cropped and rescaled to $112 \times 92$ whose gray levels ranged between 0 and 255 .

In our experiments, the first $l$ images ( $l$ varies from 4 to 7) of each individual are selected for training, while the remaining images are used for testing in ORL database. Fig. (3) demonstrates the recognition rates of FDLPP algorithm over the variance of the dimensionality of subspaces when the first five images per class are selected for training. For each $l$, Fig. (2) shows the recognition rates of FBWSC. Fig. (3) shows the recognition rates over the variance of different number of classes. Fig. (4) demonstrates the recognition rates over the variance of different number of classes. The performance of FBWSC is better than 2DLDA, RC2DPCA and FBMMC.

\section{B. Experiments on the FERET Database}

The FERET face database [14] contains 14,126 images from 1199 individuals. In our experiments, we select a subset which contains 1400 images of 200 individuals (each individual has seven images). The subset involves variations in facial expression, illumination and pose. In our experiments, each image in FERET database is manually cropped and resized to $80 \times 80$.

In the experiments, $l$ images ( $l$ varies from 3 to 5$)$ are selected from the image gallery of each individual to form the training sample set. The remaining images are used for testing. Fig. (5) showed the variation of accuracy along different number of eigenvectors used and the recognition accuracy when the first four images per class are selected for training. Fig. (6) shows the recognition rates of FBWSC over the variance of different value of $l$ on the FERET database. Fig. (7) demonstrates the recognition rates over the variance of different number of classes. 


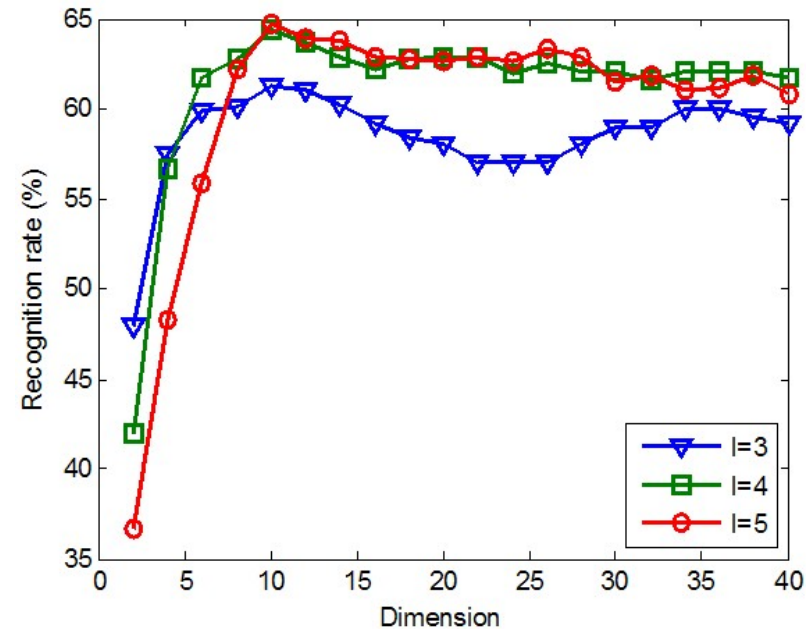

Fig. (7). Recognition rates over the variance of $l$ on FERET.

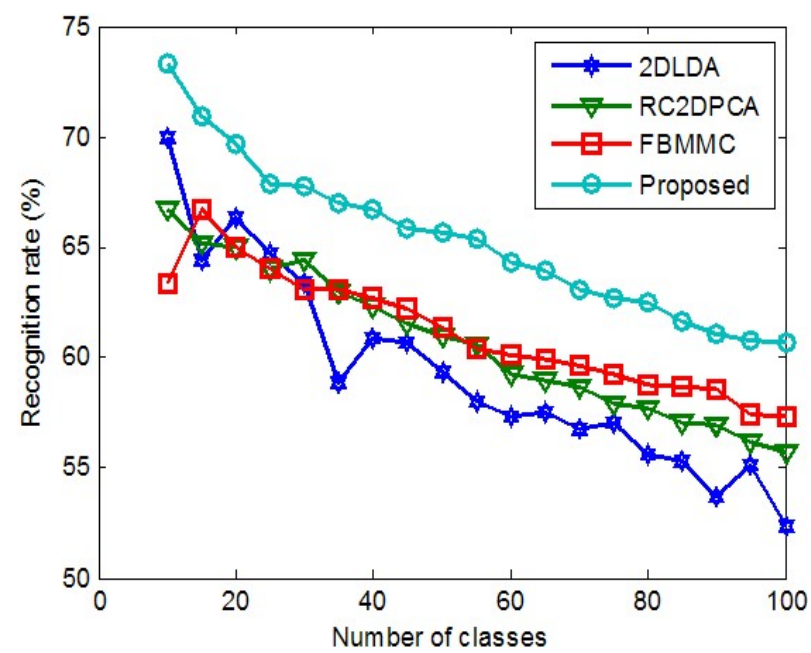

Fig. (8). Recognition rates over the variance of classes on FERET.

\section{Experiments on the Yale Database}

The Yale face database (http://cvc.yale.edu/projects /yalefaces/yalefaces.html) contains 165 gray scale images of 15 individuals, each individual has 11 images. The images demonstrate variations in lighting condition, facial expression (normal, happy, sad, sleepy, surprised, and winking). The images in the database are manually cropped and rescaled to $100 \times 100$.

In the experiments, $l$ images ( $l$ varies from 4 to 7 ) are selected from the image gallery of each individual to form the training sample set. The remaining images are used for testing. Fig. (8) shows the variation of accuracy along different number of eigenvectors used and the recognition accuracy when the first six images per class are selected for training. Fig. (9) demonstrates the recognition rates of FBWSC over the variance of different value of $l$ on the Yale database.

\section{Disscussion}

The above experiments reveal a number of points:

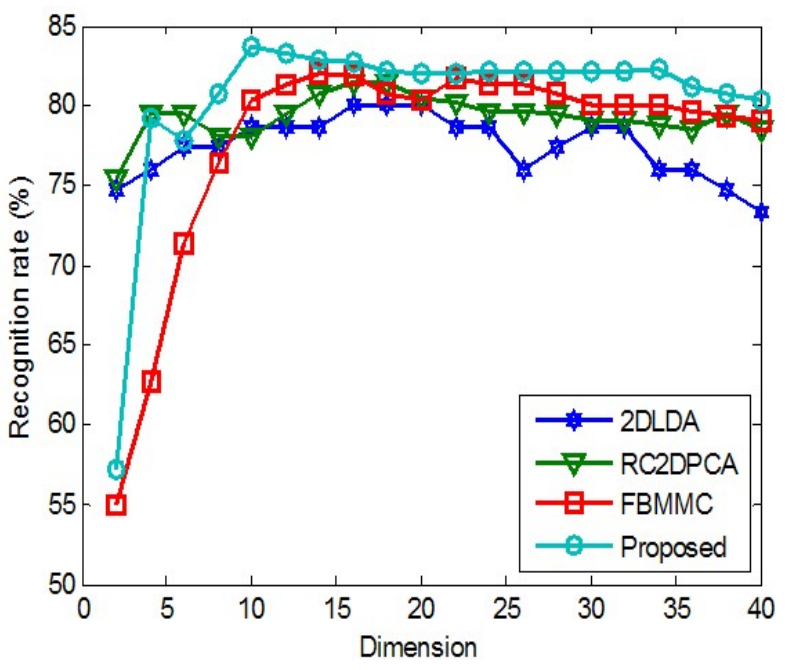

Fig. (9). The variation of accuracy along different number of eigenvectors on Yale.

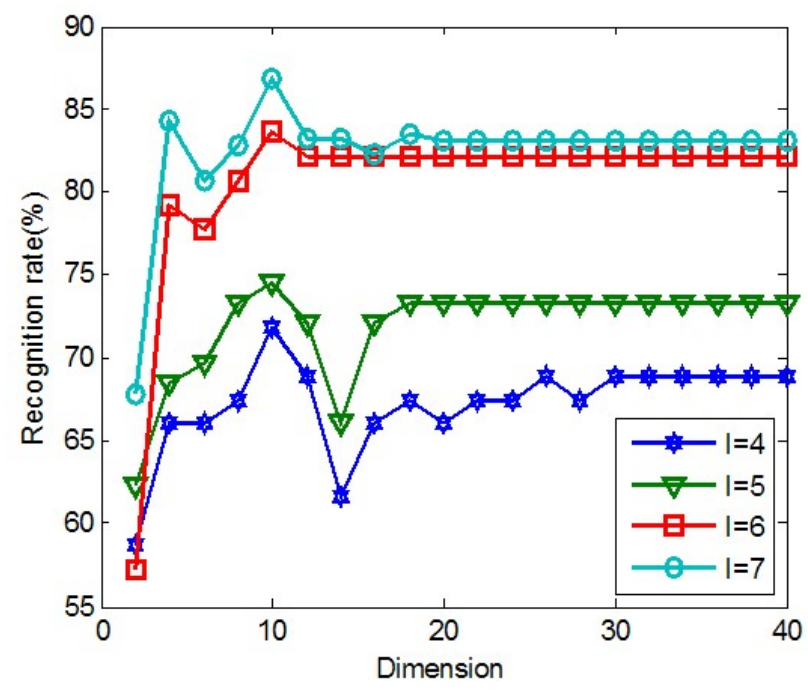

Fig. (10). Recognition rates over the variance of $l$ on Yale.

1) From Fig. (3), Fig. (6) and Fig. (9) the average recognition rate obtained by FBWSC method is higher than other methods in general and the maximal average recognition rates of all methods increase with the increase in training sample size. However, Table $\mathbf{1}$ shows that the top recognition rates of the proposed FBWSC method corresponding to the dimension is the smallest. The performance of FBWSC is better than 2DLDA, RC2DPCA and FBMMC.

2) Fig. (5) and Fig. (8) shows the accuracies decrease with the increase of the number of classes and the performance is well when the size of training is small. This demonstrates that the proposed FBWSC is avoiding the SSS problem adequately.

3) From Fig. (4), Fig. (7) and Fig. (10), we can find that the maximal average recognition rates of all methods increases with the increase in training sample size. These indicate that the proposed FBWSC is more 
suitable for reflecting the neighborhood structure of these databases.

4) From Table 1, the FBWSC method gains $2.11 \%, 5.44 \%$ and $1.27 \%$ improvement in recognition rate in ORL, FERET and Yale database, respectively. It is likely that the FBWSC method is more robust against variations in facial expression, illumination and pose.

\section{CONCLUSION}

In this paper, we present an FBWSC method for dimensionality reduction and application to face recognition. The row directional and column directional fuzzy image optimal image projection matrix are defined by FBWSC. Each sample in the original training sample set is transformed using the row and column directional optimal image projection matrix respectively. The weight on row and column directional can be calculated through the fuzzy set theory. Extensive experiments completed on ORL, FERET and Yale face databases show improved classification rates and reduced sensitivity to variations between face images caused by changes in illumination and viewing directions.

\section{CONFLICT OF INTEREST}

The author confirms that this article content has no conflict of interest.

\section{ACKNOWLEDGEMENTS}

The authors would like to thank the support of the Natural Science Foundation of Gansu Province (1212RJZA029) and the Research Foundation of Lanzhou University of Technology (0914ZX136) in China.

\section{REFERENCES}

[1] M. Turk, and A. Pentland. "Face recognition using eigenfaces". Proceeding of IEEE Conference on Computer Vision and Pattern Recognition, Maui, HI, USA, 1991: 586-591.
[2] R. O. Duda, and P. E. Hart, "Pattern Classification and Scene Analysis", New York, Wiley, 1973.

[3] S.J. Raudys, and A.K. Jain, "Small sample size effects in statistical pattern recognition: recommendations for practitioners", IEEE Trans. Pattern Anal. Mach. Intell., vol. 13, no. 3, pp. 252-264, 1991.

[4] D. Swets, and J. Weng, "Using discriminant eigenfeatures for image retrieval, IEEE Trans. Pattern Anal. Mach. Intell.,", vol. 18, no. 8, pp. 831-836, 1996.

[5] H. S. Du, M. Li, and F. Zhang, "Fuzzy bidirectional maximum margin criterion based face recognition", Chinese J. Sci. Instrument, vol. 32, no. 5, pp. 1077-1082, 2011.

[6] W. H. Li, Y. Y. Jiang, and Y. Wang, "A face recognition algorithm using a fusion method based on resampling bidirectional 2DLDA", Acta. Electron. Sin., vol. 39, no. 11, pp. 2526-2533, 2011.

[7] J. Yang, and D. Zhang, "Two-dimensional PCA: a new approach to appearance-based face representation and recognition", IEEE Trans. Pattern Anal. Mach. Intell., vol. 26, no. 1, pp. 131-137, 2004.

[8] W. K. Yang, C. Y. Sun, and R. Karl, "Sequential Row-Column 2DPCA for face recognition", Neural Comput. Appl., vol. 21, no. 7, pp. 1729-1735, 2012.

[9] W. Zuo, D. Zhang, and K. Wang, "Bidirectional PCA with assembled matrix distance metric for image recognition", IEEE Trans. Syst. Man. Cybern-Part B, vol. 36, no. 4, pp. 862-872, 2006.

[10] W. Zuo, D. Zhang, and K. Wang, "BDPCA plus LDA: a novel fast feature extraction technique for face recognition", IEEE Trans. Syst. Man. Cybern-Part B, vol. 36, no. 4, pp. 946-952, 2006.

[11] K. C. Kwak, and W. Pedrycz, "Face recognition using a fuzzy fisherface classifier", Pattern Recog. J. Patt. Recogn. Soc., Pattern Recogn., vol. 38, no. 10, pp. 1717-1732, 2005.

[12] W. K. Yang, X. Y. Yan, and L. Zhang, "Feature extraction based on fuzzy 2DLDA", Neurocomputing, vol. 73, no.10-12, pp. 1556$1561,2010$.

[13] AT\&T Laboratories Cambridge, "ORL face database", [2008-0512], http://www.camor1.co.uk/facedatabase.htm.

[14] P. J. Phillips, H. Wechsler, and J. Huang, "The FERET database and evaluation procedure for face recognition algorithms", Image Vis. Comput., vol. 16,no. 5, pp. 295-306, 1998.

Received: September 22, 2014

Revised: November 03, 2014

Accepted: November 06, 2014

(C) Lu and Jiang; Licensee Bentham Open.

This is an open access article licensed under the terms of the Creative Commons Attribution Non-Commercial License (http://creativecommons.org/licenses/by-nc/3.0/) which permits unrestricted, non-commercial use, distribution and reproduction in any medium, provided the work is properly cited. 